\title{
Hematological cellular alterations in plateletpheresis donors
}

\author{
Hanan Mahmoud Fayed, Sanaa Shaker Ali, Eman Salah Eldin
}

\begin{abstract}
Aims: Blood contact with foreign surfaces in the apheresis systems may activate many cell types that return back to the donor. However, the safety issue regarding post-procedure platelet activation is not well assessed. We aimed to evaluate the alterations in hematological parameters and to explore the formation of platelets leukocytes aggregates (PLAs) and/or complexes in healthy donors who underwent first-time plateletpheresis procedure. Methods: Blood from 100 healthy donors were assessed by BD FACS Calibur flow cytometer for: a) detection of PLAs using (CD41, CD42b, CD61) antibodies against platelets surface molecules to detect its expression on neutrophils, monocytes, lymphocytes, and b) evaluation of red cell mechanical fragility (RBC-MF). Results: After donation a significant decrement of donor blood cell counts; the percent (\%) reduction in hemoglobin (Hb) 7.9(5.1-9.2) $(p=0.017)$, hematocrit (Het) 6(2.72-7.81) $(p=0.043) \%$, residual red cells $(p=0.016)$, platelet count (PLT) 22.7(9.5-32) $(p=0.031)$ together with a significant increase in the MPV $(p=0.001)$, the absolute neutrophil $(p=0.026)$ and lymphocyte
\end{abstract}

Hanan Mahmoud Fayed ${ }^{1}$, Sanaa Shaker $\mathrm{Ali}^{2}$, Eman Salah Eldin ${ }^{3}$

Affiliations: ${ }^{1}$ Assistant Professor and Charge d'affaires, Clinical and Chemical Pathology Department, Faculty of Medicine-South Valley University, Qena, Egypt; ${ }^{2}$ Assistant Professor, Clinical and Chemical Pathology Department, Faculty of Medicine-South Valley University, Qena, Egypt; ${ }^{3}$ Lecturer, Clinical and Chemical Pathology Department, Faculty of Medicine-Assiut University, Assiut-Egypt.

Corresponding Author: Hanan Mahmoud Fayed, Qena Faculty of Medicine South Valley University 6th km Qena Safaga, Road, Qena, Egypt 82523; Email: hananfayed@yahoo. com; Drhanan.fayed@med.svu.edu.eg

Received: 02 March 2018

Accepted: 03 May 2018

Published: 13 June 2018 count $(p=0.041)$ with an insignificant increase in absolute monocyte $(p=0.103)$, lymphocyte subsets count, CD4:CD8 ratio, and WBC count. However, there was significant increase in the median platelets complexed with neutrophil, lymphocytes, and monocytes. Conclusion: All donors had a significant drop in all blood counts; none of them manifested features of thrombocytopenia or anemia. However, an increase in PLAs formation provides an evidence of ongoing platelet activation, a platelet-leucocyte interaction that may induce a pre-thrombotic risk. This result is important to consider as it might have potential therapeutic implications. Nevertheless, more prospective studies are essential to establish guidelines for donor safety.

Keywords: Donor safety, Flow cytometry, Platelet-leukocyte aggregates, Single donor platelet

\section{How to cite this article}

Fayed HM, Ali SS, Eldin ES. Hematological cellular alterations in plateletpheresis donors. Int $\mathrm{J}$ Blood Transfus Immunohematol 2018;8:100041Zo2HF2018.

$$
* * * * * * * * *
$$

Article ID: 100041Zo2HF2O18

$$
* * * * * * * *
$$

doi: 10.5348/100041Zo2HF2018OA

\section{INTRODUCTION}

The rapidly expanded clinical uses of blood component therapy resulted in great improvement in the field of therapeutic apheresis [1].

The concerns of patient care are given highest priority, therefore substantial efforts were made to support the widespread use of single donor platelet-apheresis (SDP) instead of platelet concentrate (PC), and these are mainly 


\section{EDORIUM Journals}

relevant to the care of patients with hematologic diseases [2].

Moreover; SDP has many advantages including economic use of blood due to selective components collection with the elimination of unnecessary component separation in the laboratory, the possibility of frequent donations, reduced donor exposures and hence reduced risk of alloimmunization [3].

Platelets apheresis procedures are usually safe and well tolerated. However, it can cause adverse local or systemic reactions to the donors [4].

Apheresis may cause alterations in donor's hemorheology because of anti-coagulant used, changes in blood composition and extracorporeal circulation [5].

We aimed to evaluate the alterations in cellular hematological parameters and to explore the formation of platelets leukocytes aggregates (PLAs) [platelet-monocyte complexes (PMC), platelets-neutrophil complexes (PNC), and platelets-lymphocyte complexes (PLC)] in healthy donors who underwent first-time platelet-apheresis procedure.

\section{MATERIALS AND METHODS}

\section{Study population}

A cross-sectional study involving hundred healthy first-time platelet-apheresis donors selected with the following criteria: weight $>60 \mathrm{~kg}$; age between 18 and 50 years; hemoglobin $>12.5 \mathrm{gm} / \mathrm{dl}$ and platelets count $>$ $200 \times 10^{9} / \mathrm{L}$; negative serological tests for HIV, hepatitis B surface antigen \& hepatitis C and syphilis; absence of any illness; none of them used anti-inflammatory drugs and with suitable venous accesses. All donations were performed at a tertiary care blood transfusion center of Assiut University Hospitals from March 2017 to December 2017. The study was conducted in accordance with the Helsinki Declaration as revised in 2008. Procedure details were explained to each donor who gave informed consent before the procedure. Ante-cubital veins were used for the venipuncture in all the donors. Vital signs were monitored at the beginning and end of each procedure; donors were also monitored for adverse events during the procedures.

\section{Single-donor platelet equipment}

Platelet apheresis was obtained using Fenwal Amicus $^{\mathrm{TM}}$ separator version 3.1 software; singleneedle system platelet-apheresis kit (PL 2410, Fenwal Inc.) (Fenwal, Lake Zurich, IL, USA) used as per the manufacturer's standard operating procedure with a whole blood (WB)/acid citrate dextrose (ACD-A) ratio of 11:1, interface set point 0.60 and blood flow rate 60$75 \mathrm{ml} / \mathrm{min}$. The donors' data (weight, sex, height, $\mathrm{Hb}$, and pre-apheresis PB PLT count) were entered into the cell separator program to determine the blood volume processed to reach the target platelets yield $\left(3 \times 10^{11}\right)$.

\section{Blood samples}

Five milliliter venipuncture blood sample was drawn twice from the donors; (before the beginning of the apheresis procedure and after the completion of the apheresis procedure), from a vein that was not to be used during the procedure), with minimum stasis through a 21-gauge needle into sterile evacuated tubes (BD Vacutainer, San Jose, CA) containing EDTA and $3.8 \%$ sodium citrate with a blood: anticoagulation ratio of 9:1) (to reduce art factual platelets activation due to tissue factor contamination, the first blood sample drawn was not used for flow cytometer (FCM)). All samples were processed within 10 min of collection for complete blood count (CBC), assessment of RBCs osmotic fragility assessment besides platelets activation and formation of platelet-leukocyte aggregates (PLAs) in blood [plateletmonocyte complex (PMC), platelet-neutrophil complex (PNC) and platelet-lymphocyte complex (PLC)] were determined by FACS Calibur FCM (BD Biosciences, San Jose, CA, USA); after proper setting, calibration, and compensation; data acquisition and analyzes was done by Cell Quest Pro software (v5.2).

- $\quad$ CBC by a calibrated hematology analyzer CellDyn 3500 (Abbott Diagnostics, IL-USA). Donor platelet percentage (\%) loss was calculated using the following formula: platelet loss $=$ (pre-platelet count) - (post-platelet count) $\times 100 /$ (pre-platelet count). The percent change of donor MPV $=$ the difference in the donor's pre- and post-procedure MPV $\times 100 /$ (pre-MPV).

- Lymphocyte subsets: Using whole blood samples; $2 \mathrm{ml}$ blood in EDTA vacutainer tube for CBC using cell dyne-180o (Abbott Diagnostics, USA) and analysis of peripheral blood lymphocyte using BD FACS Caliber ${ }^{\mathrm{TM}}$; (Becton Dickinson Biosciences, USA). T cell subset performed via tricolor using the following fluorescent-labeled monoclonal antibodies from Becton Dickinson (BD, San Jose, CA-USA); (CD3 [peridininchlorophyl proteins (PerCP)], CD4 [fluorescent isothyocyanate (FITC)], and CD8 [phycoerythrin $(\mathrm{PE})]$ as described by the manufacturer. Data processing was carried out using Cell quest 3.0.1 software. Acquisition of cells was standardized for 10,000 events per sample and fluorescence was measured on a logarithmic scale. A lymphocytes gate was created based on their forward and side scatter properties, i.e., size and granularity; the percentages and the absolute count of the lymphocyte subpopulations were calculated by multiplying the percentage (results of flow cytometer) by absolute lymphocyte counts (results of CBC), (Figure 1).

- Flow-cytometry Osmotic Fragility Test (FCM-OF): Done according to the process described by (Won and Suh) with a hemolysis inducing agent, distilled water (DW), was spiked 


\section{EDORIUM Journals}

Int J Blood Transfus Immunohematol 2018;8:100041Z02HF2018.

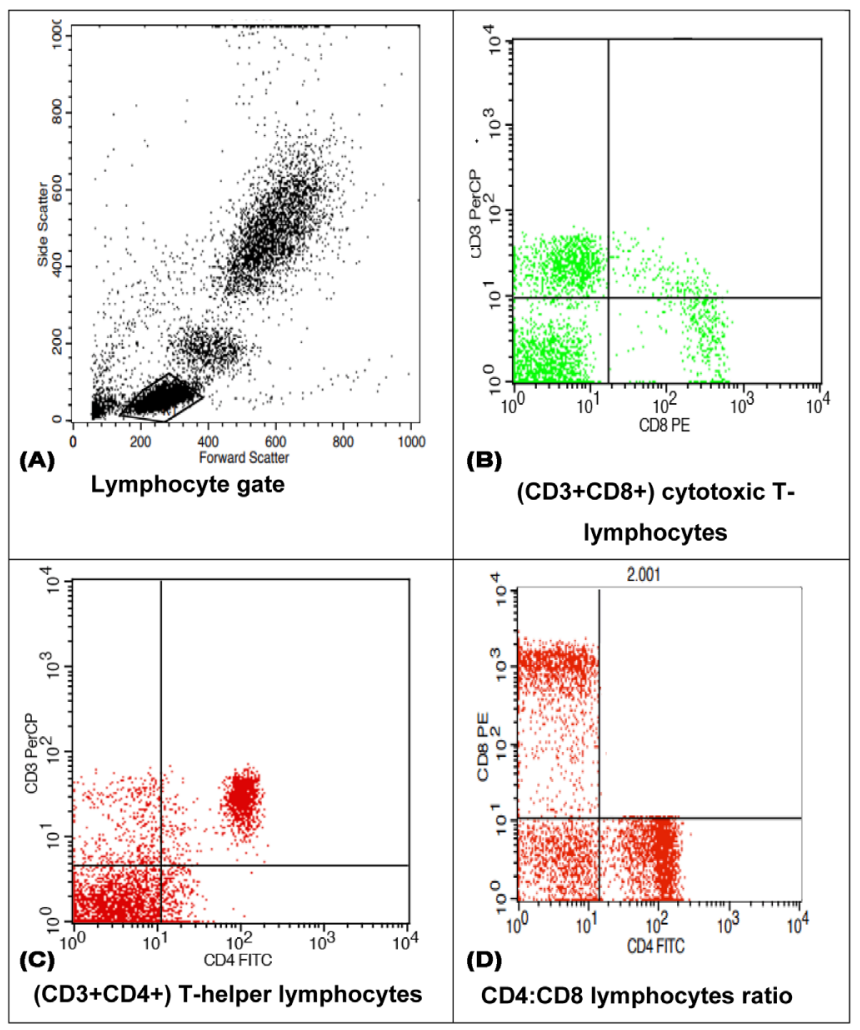

Figure 1: Dot plot analysis of peripheral blood lymphocyte subsets: (A) Lymphocyte gate; (B) Cytotoxic T-cells; (C) Helper T-cells; (D) CD4:CD8 ratio.

to a red cell suspension during acquisition, and the count of red cells was measured sequentially in real-time using FCM [6]. Acquisition performed using in low-pressure flow via linear amplification; forward scatter (FSC) and side scatter (SSC) characteristics were acquired and time/FCS acquisition plot was performed to determine with eight identical regions. The acquisition was stopped either when a 200000 event was acquired or when $102.4 \mathrm{sec}$ passed. The degree of RBCs lysis was expressed as "\% residual RBCs = mean event count of last 2 regions $\mathrm{x}$ 100 (\%) / Event count in first region x 1.1/2.0; correction factor, 1.1/2.0 is also introduced in the formula to counter the dilution in region 1 as compared to dilution after spiking with DW in other regions. The FCM-OF test was proved to be valid for screening for red cell membrane disorders [7] Figure 2 (A and B).

\section{Quality control}

To validate the operation we added normal saline for each sample instead of adding DW. Theoretically, the percentage of residual red cells will be close to $100 \%$ because the number of red cells will not change in the tube. This control checks the stability of the red cells in the suspension and excludes other causes of hemolysis other than the addition of DW. The stability of the results

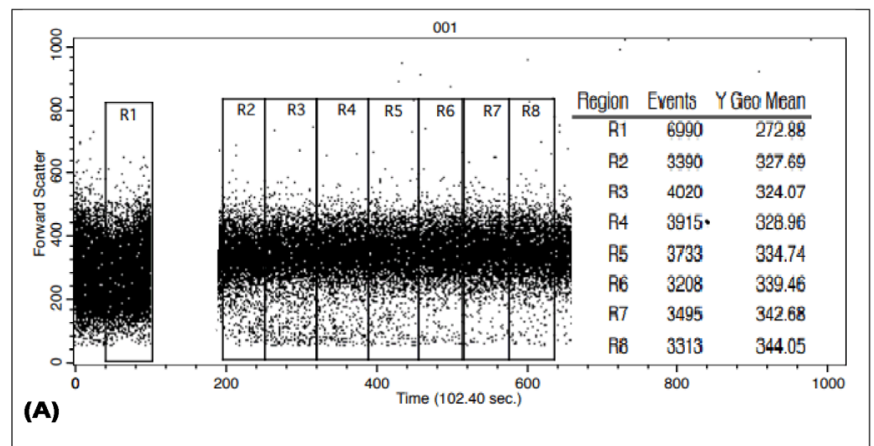

(A)

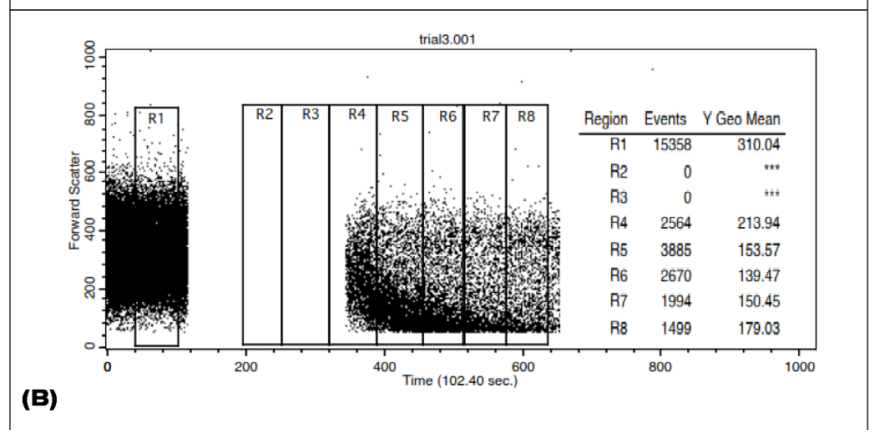

Figure 2: (A and $\mathrm{B})$ : Red cell fragility before and after platelets-apheresis procedure: (A) The FSC/time plot of red cells [\% residual red cells before and after plateletapheresis]; (B) The FSC/time plot of red cells [\% residual red cells after plateletapheresis].

over the time was determined by repeating each sample six times and calculating the coefficient of variation of the run.

\section{Leukocyte-Platelet Aggregates (PLAs)}

Monoclonal antibodies (MoAb): Either conjugated with fluoroisothiocyanate (FITC), phycoerythrin (PE) or peridinium-chlorophyll-protein (Per-CP): (CD4-PE), (CD8-PercP), (CD11c-PE), (CD19$\mathrm{PE}$ ), (CD42a-FITC), (mouse IgG-FITC) and mouse IgG-PE were purchased from Becton Dickinson, BDBiosciences, San Diego, CA, USA. (CD3-FITC), (CD41FITC) and (CD61-PE) were purchased from Beckman Coulter Immunotech (Marseille, France). For platelets glycoprotein (GP) surface markers we used [(CD41a) (GPIIb), (CD61) (GPIIIa), and (CD42a) (GPIX and GPIb-IX-V)]. (CD11c) was used for monocytes and granulocytes and MoAb mouse conjugated FITC, PE, and PercP isotype-identical MoAb were used as negative controls.

Measurements ofleukocyte-plateletaggregates (LPAs): were analyzed immediately to reduce the effects of time-dependent platelet and leukocyte activation kinetics [8]. After lysis of the erythrocytes; the FCM measurement of LPAs was performed as previously described [9]. Gating on leukocyte populations was then performed to exclude single platelets using dot plot FSC and SSC and quantification of the expression of platelets markers (CD41, CD42a, and CD61) on neutrophils, lymphocytes, and monocytes is used for 


\section{EDORIUM Journals}

quantifying of the formation LPAs (PNC, PLC, and PMC) for each leukocyte cell population and calculated as the percentage of monocytes, neutrophils and lymphocytes. For each sample, un-stained cells were used to define the "Leukocyte" gate in the dot plot scatter and the neutrophil, and monocytes were gated based on their forward and side scatter (FSC/SSC) characteristics. CD41, CD61, CD11c, CD42a, IgG1 and IgG2 expression was analyzed on the gated population. Results were recorded as percentage of the positive gated cells. The PMC, PLC, and PNC were calculated as the percentage of monocytes, lymphocytes, and neutrophils that express platelet markers, Figure 3 (A-D).

Yet, detection of platelets complexes are not very straightforward due to the fact that platelets may undergo spontaneous activation during sample processing and once activated, they can directly form complexes with surrounding cells [10].

\section{Quality control}

Daily cleaning of the flow cell fluidics line was performed using filtered FACS ${ }^{\text {тм }}$ BD Clean Solution. Antibody titrations were performed to determine the optimal antibody concentrations and color compensation was performed using the versa comp antibody capture beads (BD Biosciences, San Jose, CA, USA). An unstained fresh blood sample was used to set the voltages for the FS/ SS. Debris and electronic noise were excluded by setting the appropriate forward scatter threshold. Stained whole blood was used to determine the percentage of spectral overlap and to perform color compensation. At least

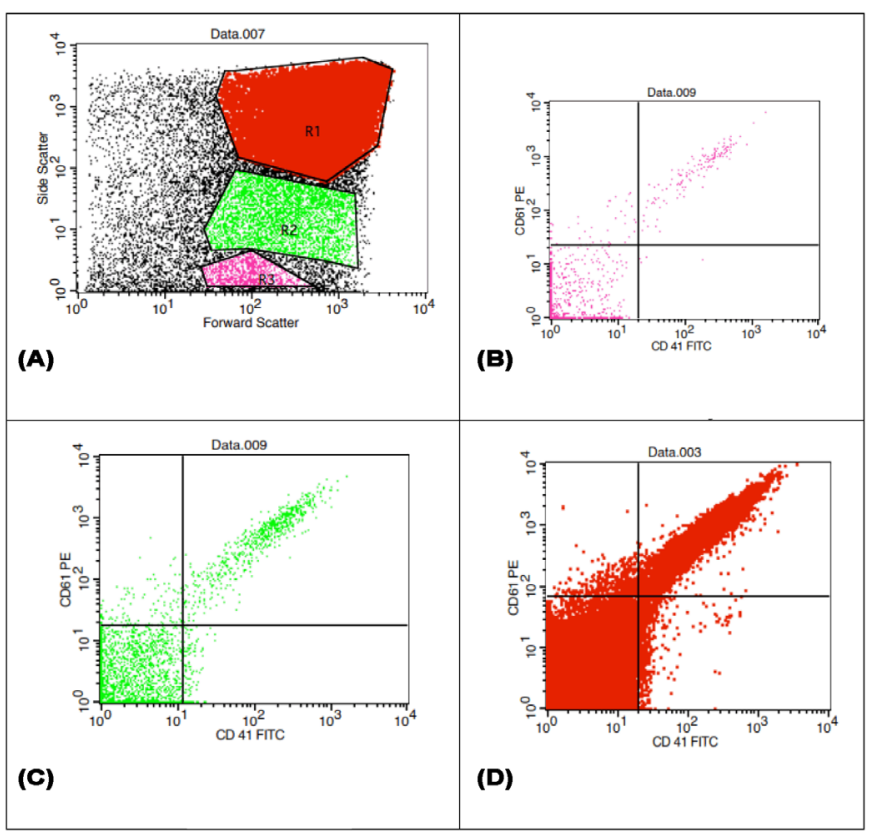

Figure 3: Forward scatter and side scatter leukocyte dot plot: (A) Gating on neutrophils, monocytes, lymphocytes; (B) CD41/ CD61 co-expression monocyte; (C) CD41/CD61 co-expression on lymphocyte; (D) CD41/CD61 co-expression on neutrophil.
100000 events were acquired at a medium flow rate to minimize coincidental events.

\section{Statistical analysis}

Shapiro-Wilk test was conducted to assess normality and for non-normal distribution; the Mann-Whitney U-test was used to compare between groups. Quantitative data were presented as median and range. Categorical data were presented as number and percentage. All tests were two-sided and a probability value of $<0.05$ was considered statistically significant. Data analysis was performed using Statistical Package for the Social Science (SPSS) version 22 software (Chicago, IL, USA).

\section{RESULTS}

A total of 100 healthy male first-time plateletapheresis donors were included in this study. There were no adverse events that cause early termination of the procedure, and no access-related complications indicating that the procedure is well tolerated by donors. However, a mild complication was recorded but does not significantly impair donor safety or product quality; consisted of hypotension (5\%) that responded rapidly to decreased flow rates. None of the donors received prophylactic calcium during the procedure.

Platelet-apheresis donors' characteristics and procedural data were presented in Table 1.

The average value of pre-donation $\mathrm{Hb}$ was $14.14 \pm 0.08$ gm $\%$ and platelet count was $305.28 \pm 71.92$ per $\mu l$. In the post-donation, the percent residual red cells, $\mathrm{Hb}$ and Hct were significantly decreased $(\mathrm{P}=0.016),(\mathrm{p}=0.017)$ and $(\mathrm{p}$ $=0.043)$ respectively, the PLT count showed a significant decrease $(p=0.031)$ together with a significant increase in the MPV ( $p=0.001)$, and significant increase in the absolute neutrophil $(\mathrm{p}=0.026)$ and lymphocyte count ( $\mathrm{p}=0.041)$ with an insignificant increase in absolute monocyte $(\mathrm{p}=0.103)$, lymphocyte subsets count, CD4:CD8 ratio and WBC count (Table 2).

In the post-donation, data showed a significant increase in the median platelet binding to neutrophils, lymphocytes, and monocytes forming PLAs; associated with an increase in the number of platelet markers (CD41, CD42a, and CD61) complexed with neutrophil lymphocytes, and monocytes (Table 3 and Figure 4 A-C).

\section{DISCUSSION}

Extracorporeal circulation activates blood cells and coagulation process through the contact of blood with artificial surfaces [11]. Even though there were no apparent harmful effects of extracorporeal circulation during platelet-apheresis, concerns have been raised that it may activate coagulation [12].

Apheresis is a relatively safe process that is commonly used for donation and therapeutic goals. The most 


\section{EDORIUM Journals}

Int J Blood Transfus Immunohematol 2018;8:100041Z02HF2018.

www.ijbti.com

Table 1: Platelet-apheresis donors' characteristics and procedural data

\begin{tabular}{lcc} 
& Median (range) & Mean \pm SD \\
\hline Age (years) & $26.3(20-42)$ & $30.69 \pm 6.35$ \\
Weight $(\mathrm{kg})$ & $82.5(65-85.5)$ & $77.32 \pm 23.53$ \\
Height $(\mathrm{cm})$ & $169(155-185)$ & $164.58 \pm 10.75$ \\
BMI $\left(\mathrm{kg} / \mathrm{m}^{2}\right)$ & $26.80(23-28)$ & $24.60 \pm 4.32$ \\
Blood volume processed (mL) & $4354(4150-5398)$ & $4617 \pm 504$ \\
Procedure time (Min) & $71.5(62-75)$ & $69.90 \pm 9.73$ \\
ACD used during procedures (mL) & $432(375-473)$ & $425 \pm 27$ \\
Donor platelets loss (\%) & $22.67(9.47-32.03)$ & $18.30 \pm 10.67$
\end{tabular}

Table 2: Pre- and Post-Apheresis Peripheral Blood cell Variables

\begin{tabular}{|c|c|c|c|c|}
\hline $\begin{array}{l}\text { Parameter } \\
\text { Median (range) }\end{array}$ & Pre-donation & Post-donation & $\%$ change & p-value \\
\hline $\mathrm{Hb}(\mathrm{g} / \mathrm{dl})$ & $14.25(12.8-15.6)$ & $13.4(11.8-15.1)$ & $-7.9(5.1-9.2)$ & $\mathbf{0 . 0 1 7 ^ { * }}$ \\
\hline Het (L/L) & $42.2(36.9-45 \cdot 7)$ & $40.26(35 \cdot 4-44$ & $-6(2.72-7.81)$ & $0.043^{*}$ \\
\hline \% residual red cells & $112.25\left(85^{-152.4}\right)$ & $90.3(60-135)$ & $-16.73(4.9-38.3)$ & $0.016^{*}$ \\
\hline Platelets count $\times 10^{9} / \mathrm{L}$ & $327(187-437)$ & $262(150-355)$ & $-22.7(9.5-32)$ & $0.031^{*}$ \\
\hline MPV (fl) & $8.1(7.1-8.9)$ & $9.4(7.5-11.5)$ & $+16(3.8-29.6)$ & o.001 ${ }^{*}$ \\
\hline WBCs $\left(\mathrm{x} 10^{9} / \mathrm{L}\right)$ & $6.600(5.470-10.400)$ & $7.150(5.800-8.900)$ & $+14.5(0.6-30.7)$ & 0.477 \\
\hline Neutrophil (x10/L) & $3.240(1.380-6.131)$ & $4.485(2.734-5.803)$ & & $0.026^{*}$ \\
\hline Lymphocyte (x109/L) & $2.855(1.529-5.408)$ & $2.249(1.170-3.434)$ & & $0.041^{*}$ \\
\hline Monocyte (x109/L) & $0.469(0.200-0.660)$ & $0.528(0.221-0.950)$ & & 0.103 \\
\hline 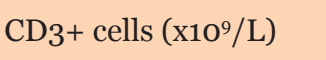 & $0.96(0.2-1.1)$ & $0.8\left(0.5^{-2}\right)$ & & 0.728 \\
\hline $\mathrm{CD} 4+$ cells $\left(\mathrm{x} 10^{9} / \mathrm{L}\right)$ & $0.46(0.17-1.1)$ & $0.7(0.4-1.6)$ & & 0.124 \\
\hline 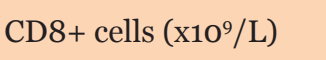 & $0.1(0.02-0.34)$ & $0.1(0.05-0.4)$ & & 0.478 \\
\hline $\mathrm{CD} 4: \mathrm{CD} 8\left(\mathrm{x} 10^{9} / \mathrm{L}\right)$ & $5 \cdot 5(1.23-11)$ & $5\left(2.5^{-16}\right)$ & & 0.174 \\
\hline
\end{tabular}

*significant: Mann-Whitney U Test

common platelet collection procedural adverse effects are citrate related such as shivering, nausea, vomiting, abdominal pain, chills, fever, lightheadedness, tremors, and muscle cramps, severe hypocalcemia that may progress to frank tetany, and grave laryngospasm [13]. The incidence of citrate toxicity varies from 0.11 to $16 \%$ [14]. The predictors of citrate toxicity include older age, female, low body weight, frequent apheresis, and longer total time for donation. In addition, the amount of reinfused citrate is influenced by the efficiency of centrifugation separation, the return speed, amount and type of citrated blood component returned [15].

In this study, we found a significant reduction of postdonation platelets count (but remained clear within the normal range), a significant fall in $\mathrm{Hb}$, Hct and RBCs but insignificant increase of WBC count. This was partially consistent with several studies [16-24] who reported a clinically insignificant fall in the donors' hematological parameters after platelet-apheresis; as a consequence of many factors such as haemodilution due to citrate and saline infusions [25]; reduction of platelet count, probably beside platelets collection; it might also be due to adhesion of platelet to tubing or centrifuge bowl [4], besides adherence of WBCs and RBCs to the surfaces of the extracorporeal circulation tubes [16]. Likewise; the blood loss in the void volume of the apheresis kit, the technique applied and mechanical hemolysis by the pressure pumps [26]. 
Table 3: Platelet leukocyte aggregates

Parameters Median (range)

Platelet-Neutrophil Complexes (PNC)

CD11c+ neutrophils

$\mathrm{CD} 42 \mathrm{a}+$ neutrophils

$\mathrm{CD} 11 \mathrm{c}+\mathrm{CD} 42 \mathrm{a}+$ neutrophils

CD41+ neutrophils

CD61+ neutrophils

CD41+ CD61+ neutrophils

$\mathrm{IgG} 1+$ neutrophils

IgG2+ neutrophils

IgG1 + IgG2a + neutrophils

IgG2a+CD41+ neutrophils

Platelet-lymphocytes Complexes

CD42a+lymphocytes

CD41+lymphocytes

CD61+ lymphocytes

CD41+ CD61+ lymphocytes

Platelet-Monocyte Complexes (PMC)

CD11c+ monocytes

CD42a+ monocytes

$\mathrm{CD} 11 \mathrm{c}+\mathrm{CD} 42 \mathrm{a}+$ monocytes

CD41+ monocytes

CD61+ monocytes

CD41+ CD61+ monocytes

IgG1+ monocytes

IgG2+ monocytes

IgG1+ IgG2a+monocytes

IgG2a+CD41+ monocytes
Pre-donation

Post-donation

p-value*

$$
\begin{gathered}
44(4-63.5) \\
22.4(10-33) \\
12.5(0.6-18.7 \\
20(11-31.5) \\
25(11-40.9) \\
17(10.5-28.63) \\
12(2-35) \\
69(43.5-83.8) \\
10(1.9-28.08) \\
18.3(10.17-25.15)
\end{gathered}
$$

$42.5(12-57)$
$35(17.5-50)$
$12.57(10-29.66)$
$32(14-50.3)$
$30(13-54.7)$
$30(10.5-49)$
$19(9-28)$
$72(52-77)$

0.803

$0.005^{*}$

0.124

$0.011^{*}$

0.174

0.002 *

0.085

0.535

16.25(7.62-20.76)

$0.037^{*}$

$0.014^{*}$

22.32(14-30.49)

18.5(9.7-79.7)

$0.003^{*}$

9.5(4-17.5)

12(5-19)

14(6-25)

18.2(6-85)

0.056

$23.7(7.3-87)$

0.085

11(4.76-17.1)

18.2(5.4-85)

$0.014^{*}$

1.2(0.2-26)

5.5(2-14)

$0.005^{*}$

16.5(5.8-23)

24.5(14.5-32)

$0.023^{*}$

$0.4(0.04-7.6)$

2.2(0.7-5.42)

$0.004^{*}$

14(9.7-18)

21.3(14-34.3)

$0.003^{*}$

18.5(8-30)

11.8(7-17.46)

$35.6(18.5-43.6)$

$0.001^{*}$

22(9.09-33)

$0.0004^{*}$

2.4(0.6-7.5)

8.3(0.9-42.8)

0.48(0.06-3)

2.64(1.47-7.46)
6.5(2.2-15.3)

$0.0139^{*}$

37(9.7-53)

$0.005^{*}$

4(0.58-9.8)

$0.001^{*}$

7.94(3-14.41)
0.0004 *

* Mann-Whitney U Test, significant at < 0.05

In this study; there was post donation reduction of platelet count $22.7 \%$, this was in line with Beyan and his collages reported that the mean reduction in PLT on Amicus was 31.9\% [25] and Kalish and coworkers found a 29.4\% decrease in PLT count after donation [19]. However; Gilcher and Smith reported that nearly 25$50 \%$ of circulating platelets might be lost during single platelet-apheresis but this is usually normalized by the spleen, Hence, the clinical findings of thrombocytopenia are unusual in donors [4].

In contrast, Sachdeva and coworkers revealed postdonation increase in $\mathrm{Hb}$ and Hct due to back transfusion of concentrated red cells to the donor and the plasma kept to the end of the procedure, and the blood sample was taken immediately after the procedure [27], while Mahmood and his collages and Patidar and his coworkers found post-donation increase in WBC with significant reduction of the mean post-donation values for $\mathrm{Hb}$, Hct, PLT, MPV, and PDW [22-29].

However, Das and his collages reported post-donation thrombocytopenia in nearly $36 \%$ of the healthy donors who had a pre-donation platelet count of $<200 \times 10^{9} / \mathrm{L}$ [29]. Also Suresh and coworkers reported a decrease in platelets count together with an increase in PDW [23]. But Sachdeva and his collages found a 30.7\% decrease in post-donation platelets count and insignificant decrease in the MPV and in PDW [27].

In this study, a reduction of $\mathrm{Hb}$, and platelets were $7.9 \%$ and $22.7 \%$ respectively. Yet; Tendulkar and Rajadhyaksha found a reduction in the $\mathrm{Hb}$, and platelets were 2.9 and $30.7 \%$ respectively [24]; and Das and coworkers found $1.3 \%$ reduction of $\mathrm{Hb}$, and $61.6 \%$ of platelets [17].

In this study, we found a significant increase of MPV, which may be sign reflecting subclinical platelet activation. In contrast; Tenorio et al found significant reduction of MPV this could be due to the shorter process time in their study [30].

In this study, we found a post-donation significant increase in the absolute neutrophil and significant the absolute decrease in lymphocyte count with an 


\section{EDORIUM Journals}

Int J Blood Transfus Immunohematol 2018;8:100041Z02HF2018. www.ijbti.com
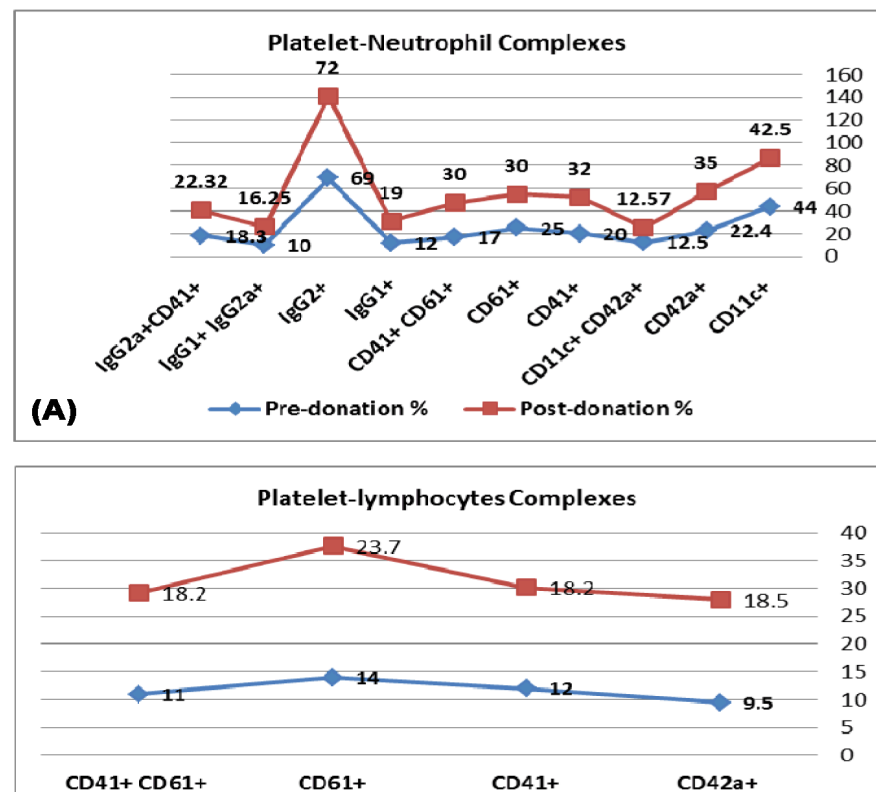

(B) $\multimap$ Pre-donation $\% \quad \rightarrow-$ Post-donation $\%$

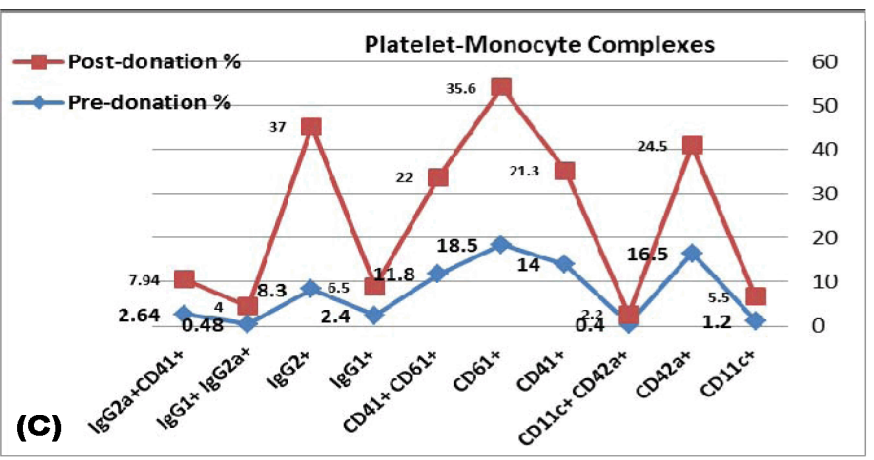

Figure 4 (A-C): Platelets-leukocytes complexes pre- and postdonation: (A) Platelet- neutrophils complexes; (B) Plateletslymphocytes complexes; (C) Platelets-monocyte complexes.

insignificant increase in absolute monocyte, lymphocyte subsets count, and WBC count. This was partially in agreement with Western and Videm reported an insignificant change in the donors mean leukocyte counts [31]. In contrast; Prior and coworkers found a transient drop in the absolute lymphocyte counts and $\mathrm{T}$ cells in healthy cytapheresis donors (mostly regular donors) during cardiopulmonary bypass [32]. However, Rinder and his collages found that the alteration of lymphocyte count was normalized within 3 days after the extracorporeal procedure [33].

In this study, we found that the expression of (CD11c \& CD42a), (CD61), (CD11c), (IgG1) and (IgG2) postdonation was significantly increased on monocytes but with insignificant changes in the expression of (CD11c \& CD42a), (CD61), and (CD11c) on neutrophil. However, there were significant increase in post donation expression of (IgG1 \& IgG2a) and (IgG2a \& CD41) on both monocytes and neutrophil, this was in agreement with Western and Videm [31] who found slight neutrophil function change after apheresis owing to contact between neutrophils and the apheresis foreign surface found to be a bio-tolerant procedure and also in agreement with Ahn and coworkers who established that activated platelets bind preferably to monocytes to form the plateletmonocyte complex (PMCs) [34]. This can be explained by the fact that during the apheresis procedure, the donor blood was exposed to an artificial membrane and external forces such as centrifugation that might activate platelets and leukocytes [35]. Upon activation; platelets undergo granule exocytosis and expression of p-selectin on the cell membrane which binds to a counter-receptor ligand expressed on leukocytes forming PLAs [34], with their subsequent clearance either by the phagocytic system or by the trapping in the microcirculation [36]. It was considered that the number of circulating PMCs is a sensitive marker of platelet activation than P-selectin expression because degranulated platelets aggregate with leukocytes in vitro and lose surface p-selectin rapidly in vivo [10].

In this study, we found post-donation significant increase in platelets antigen expression (CD41, CD42a, and CD61) on the leukocytes, signifying PLAs and formation of complexes with (monocytes, lymphocytes, and neutrophils) formation. This was in line with [37].

Jung et al. suggested that the separation process could be responsible for platelet activation as result from the different traumatization of platelets [38]; and it was established that the activated platelets play a key role in the initiation of both inflammation and coagulation [39].

Thus our findings may provide evidence that platelets activation occurs during apheresis and the activated platelets continue to circulate with the formation of the platelet-leukocyte complex; and so may induce a prethrombotic risk. Comparable approaches, to protect PLTs from activation e.g., during cardiopulmonary bypass (CPB) were tested in experimental studies with baboons. Temporary inhibition of PLT function "PLT anesthesia" during CPB with a reversible inhibitor of PLT glycoprotein IIb/IIIa (Tirofiban) improved PLT function after bypass and accelerated restoration of normal template bleeding time [40]. And Zeiler et al found that the ingestion of 500 mg Aspirin ASA 12 hours before the apheresis resulted in a significant decrease of donor's induced PLT aggregation without harmful effects to the stored product clinical efficiency [41].

Limitation of the study: The use of resting platelets markers; not an activation-dependent platelets marker, short time follow-up for donors after the procedure of plateletpheresis.

\section{CONCLUSION}

The present study raised donor safety regarding the post-donation drops in hematological values. Although such reductions could be expected, adverse clinical outcomes, such as thrombocytopenia and anemia, should always be prevented by proper donor selection, 
and careful monitoring to settle down donor safety and quality programs for a healthy quality assurance.

We revealed that in healthy donors during apheresis platelets activation occurs, and the activated platelets continue to circulate with the formation of the plateletleukocyte complex; and so may induce a pre-thrombotic risk, especially in those who submit to repeated plateletapheresis and/or have additional risk factors for thromboembolic events.

\section{RECOMMENDATION}

Donors with significant decrements should be reviewed later to exclude or, if necessary, treat iatrogenic anemia and thrombocytopenia. More prospective studies are required to establish guidelines for donor safety.

\section{REFERENCES}

1. Cojocari E. Apheresis nursing: ACanadian perspective. Transfus Apher Sci 2015 Feb;52(1):137-40.

2. Ness PM, Campbell-Lee SA. Single donor versus pooled random donor platelet concentrates. Curr Opin Hematol 2001 Nov;8(6):392-6.

3. Ness P, Braine H, King K, et al. Single-donor platelets reduce the risk of septic platelet transfusion reactions. Transfusion 2001 Jul;41(7):857-61.

4. Gilcher RO, Smith JW. Apheresis: Principles and technology of hemapheresis. Chapter 41. In: Simon TL, Snyder EL, Stowell CP, Strauss RG. Solheim BG, Petrides M, editors. Rossi's Principles of Transfusion Medicine. 4ed. Oxford, UK: Wiley-Blackwell; 2009. p. 617-28.

5. Bor-Kucukatay M, Keskin A, Akdam H, et al. Effect of thrombocytapheresis on blood rheology in healthy donors: Role of nitric oxide. Transfus Apher Sci 2008 Oct;39(2):101-8.

6. Won DI, Suh JS. Flow cytometric detection of erythrocyte osmotic fragility. Cytometry B Clin Cytom 2009 Mar;76(2):135-41.

7. Warang P, Gupta M, Kedar P, Ghosh K, Colah R. Flow cytometric osmotic fragility: An effective screening approach for red cell membranopathies. Cytometry B Clin Cytom 2011 May;8o(3):186-90.

8. Harrison P, Mackie I, Mumford A, et al. Guidelines for the laboratory investigation of heritable disorders of platelet function. Br J Haematol 2011 Oct;155(1):3044 .

9. Nkambule BB, Davison G, Ipp H. Platelet leukocyte aggregates and markers of platelet aggregation, immune activation and disease progression in HIV infected treatment naive asymptomatic individuals. J Thromb Thrombolysis 2015 Nov;40(4):458-67.

10. Michelson AD, Barnard MR, Krueger LA, Valeri CR, Furman MI. Circulating monocyte-platelet aggregates are a more sensitive marker of in vivo platelet activation than platelet surface P-selectin: Studies in baboons, human coronary intervention, and human acute myocardial infarction. Circulation 2001 Sep 25;104(13):1533-7.
11. Reverter JC, Escolar G, Sanz C, et al. Platelet activation during hemodialysis measured through exposure of p-selectin: Analysis by flow cytometric and ultrastructural techniques. J Lab Clin Med 1994 Jul;124(1):79-85.

12. Stohlawetz P, Kapiotis S, Seidl D, et al. Safety issues of plateletpheresis: Comparison of the effects of two cell separators on the activation of coagulation, fibrinolysis, and neutrophils and on the formation of neutrophil-platelet aggregates. Transfusion 1999 Apr;39(4):420-7.

13. Moog R, Zeiler T, Heuft HG, et al. Collection of WBCreduced single-donor PLT concentrates with a new blood cell separator: Results of a multicenter study. Transfusion 2003 Aug;43(8):1107-14.

14. Bueno JL, Barea L, García F, Castro E. A comparison of PLT collections from two apheresis devices. Transfusion 2004 Jan;44(1):119-24.

15. Lee G, Arepally GM. Anticoagulation techniques in apheresis: From heparin to citrate and beyond. J Clin Apher 2012;27(3):117-25.

16. Akay OM, Akin E, Mutlu F, Gulbas Z. The effects of plateletpheresis on donor platelet function and coagulation. Transfus Apher Sci 2007 Oct;37(2):1134.

17. Das SS, Chaudhary R, Verma SK, Ojha S, Khetan D. Pre- and post- donation haematological values in healthy donors undergoing plateletpheresis with five different systems. Blood Transfus 2009 Jul;7(3):18892.

18. Farahat MH, Sharaf MA. Hematological and biochemical parameter alteration after plateletpheresis donation. Egypt $J$ Haematol 2016;41(4):174-9.

19. Kalish RI, Chambers LA, Linden JV. The effect of plateletpheresis on the Fenwal CS-3000 on donor platelet counts. J Clin Apher 1987;3(4):230-4.

20. Keklik M, Eser B, Kaynar L, et al. Comparison of plateletpheresis on the Fenwal Amicus, Fresenius COM.TEC, and Trima Accel Cell separators. J Clin Apher 2015 Jun;30(3):171-5.

21. Lazarus EF, Browning J, Norman J, Oblitas J, Leitman SF. Sustained decreases in platelet count associated with multiple, regular plateletpheresis donations. Transfusion 2001 Jun;41(6):756-61.

22. Mahmood W, Rifin N, Iberahim S, Mastazamin LT, Mustafa R. Significant reduction in hematological values after plateletpharesis: Clinical implication to the donor. Asian Biomedicine 2011;5(3):393-5.

23. Suresh B, Arun R, Yashovardhan A, Deepthi K, Sreedhar Babu KV, Jothibai DS. Changes in preand post-donation haematological parameters in plateletpheresis donors. J Clin Sci Res 2014;3:85-9.

24. Tendulkar A, Rajadhyaksha SB. Comparison of plateletpheresis on three continuous flow cell separators. Asian J Transfus Sci 2009 Jul;3(2):73-7.

25. Beyan C, Cetin T, Kaptan K, Nevruz O. Effect of plateletpheresis on complete blood count values using three different cell separator systems in healthy donors. Transfus Apher Sci 2003 Aug;29(1):45-7.

26. Méndez A, Wägli F, Schmid I, Frey BM. Frequent platelet apheresis donations in volunteer donors with hemoglobin < $125 \mathrm{~g} / \mathrm{l}$ are safe and efficient. Transfus Apher Sci 2007 Feb;36(1):47-53. 


\section{EDORIUM Journals}

27. Sachdeva P, Kaur G, Basu S, Tahlan A. Assessment of factors affecting the platelet yield using continuous flow cell separator. Int J Biomed Res 2014;5(3):1969.

28. Patidar GK, Sharma RR, Marwaha N. Frequency of adverse events in plateletpheresis donors in regional transfusion centre in North India. Transfus Apher Sci 2013 Oct;49(2):244-8.

29. DAS SS, Chaudhary RK, Shukla JS. Factors influencing yield of plateletpheresis using intermittent flow cell separator. Clin Lab Haematol 2005 Oct;27(5):316-9.

30. Tenorio GC, Strauss RG, Wieland MJ, Behlke TA, Ludwig GA. A randomized comparison of plateletpheresis with the same donors using four blood separators at a single blood center. J Clin Apher 2002;17(4):170-6.

31. Western KH, Videm V. Donor neutrophil function after plateletpheresis. Transfusion 2000 Nov;40(11):14148.

32. Prior CR, Coghlan PJ, Hall JM, Jacobs P. In vitro study of immunologic changes in long-term cytapheresis donors. J Clin Apher 1991;6(2):69-76.

33. Rinder CS, Mathew JP, Rinder HM, Tracey JB, Davis E, Smith BR. Lymphocyte and monocyte subset changes during cardiopulmonary bypass: Effects of aging and gender. J Lab Clin Med 1997 Jun;129(6):592-602.

34. Ahn KC, Jun AJ, Pawar P, et al. Preferential binding of platelets to monocytes over neutrophils under flow. Biochem Biophys Res Commun 2005 Apr 1;329(1):345-55.

35. Gutensohn K, Alisch A, Krueger W, Kroeger N, Kuehnl P. Extracorporeal plateletpheresis induces the interaction of activated platelets with white blood cells. Vox Sang 2000;78(2):101-5.

36. Rinder HM, Bonan JL, Rinder CS, Ault KA, Smith BR. Activated and unactivated platelet adhesion to monocytes and neutrophils. Blood 1991 Oct 1;78(7):1760-9.

37. Bilgin AU, Karadogan I, Yilmaz FG, Undar L. Double dose plateletpheresis by continuous and intermittent flow devices increases platelet-neutrophil complex formation in healthy donors without noticeable neutrophil activation. Transfus Apher Sci 2007 Feb;36(1):31-7.

38. Jung F, Bläsi U, Radtke $\mathrm{H}$, et al. Plateletpheresisinduced increase in platelet reactivity using different cell separators. Infusionsther Transfusionsmed 1995 Aug;22(4):237-43.

39. Levi M, van der Poll T. Two-way interactions between inflammation and coagulation. Trends Cardiovasc Med 2005 Oct;15(7):254-9.

40. Hiramatsu Y, Gikakis N, Anderson HL 3rd, et al. Tirofiban provides "platelet anesthesia" during cardiopulmonary bypass in baboons. $\mathrm{J}$ Thorac Cardiovasc Surg 1997 Jan;113(1):182-93.
41. Zeiler T, Gritzka D, Karger R, Kretschmer V. The effect of ASA on platelet activation during apheresis and on in-vitro properties of stored platelet concentrates. Transfusion 2004 Sep;44(9):1300-5.

$* * * * * * * * *$

\section{Acknowledgements}

All the technicians in blood transfusion center at a tertiary care hospital of Assiut University Hospitals for their contribution to this work.

\section{Author Contributions}

Hanan Mahmoud Fayed - Substantial contributions to conception and design, Acquisition of data, Analysis and interpretation of data, Drafting the article, Revising it critically for important intellectual content, Final approval of the version to be published

Sanaa Shaker Ali - Substantial contributions to conception and design, Acquisition of data, Analysis and interpretation of data, Drafting the article, Revising it critically for important intellectual content, Final approval of the version to be published

Eman Salah Eldin - Substantial contributions to conception and design, Acquisition of data, Analysis and interpretation of data, Drafting the article, Revising it critically for important intellectual content, Final approval of the version to be published

\section{Guarantor of Submission}

The corresponding author is the guarantor of submission.

\section{Source of Support}

None

\section{Consent Statement}

Written informed consent was obtained from the patient for publication of this study.

\section{Conflict of Interest}

Authors declare no conflict of interest.

\section{Copyright}

(C) 2018 Hanan Mahmoud Fayed et al. This article is distributed under the terms of Creative Commons Attribution License which permits unrestricted use, distribution and reproduction in any medium provided the original author(s) and original publisher are properly credited. Please see the copyright policy on the journal website for more information. 
Access full text article on other devices

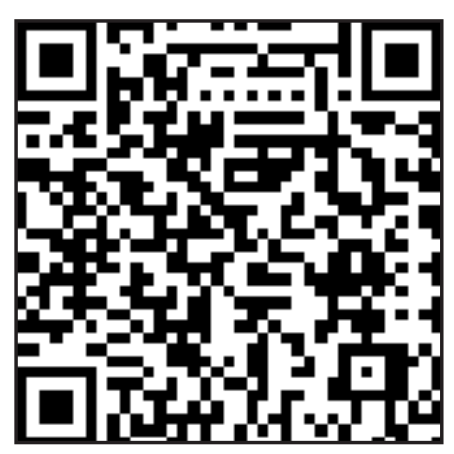

Access PDF of article on other devices

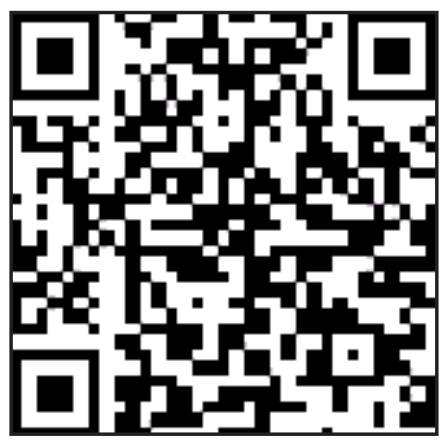

